\title{
ASPECTOS EPIDEMIOLÓGICOS DAS HEPATITES VIRAIS
}

\author{
EPIDEMIOLOGICALASPECTS OF VIRAL HEPATITIS
}

Afonso Dinis Costa Passos

Docente. Departamento de Medicina Social. Faculdade de Medicina de Ribeirão Preto - USP.

CorRespondênCIA: Avenida Bandeirantes, 3900. CEP 14049-900 Ribeirão Preto, SP. email: apassos@fmrp.usp.br

PASSOS ADC. Aspectos epidemiológicos das hepatites virais. Medicina, Ribeirão Preto, 36: 30-36, jan./mar. 2003.

RESUMO: Apresentam-os, neste trabalho, algumas das principais características epidemiológicas dos cinco tipos reconhecidos de hepatites virais, com ênfase nas causadas pelos vírus $\mathrm{B}$ e $\mathrm{C}$. Por sua larga distribuição geográfica e capacidade de causar infecções crônicas - as quais se associam a risco elevado de cirrose hepática e hepatocarcinoma - essas duas formas representam grave problema sanitário em praticamente todos os países do mundo. Discutimos sua relevância epidemiológica e os principais mecanismos de transmissão, identificados para ambas as formas.

UNITERMOS: Hepatite Viral Humana. Hepatite. Epidemiologia.

Mesmo apresentando variações importantes de incidência e prevalência, de acordo com a região geográfica, as hepatites virais representam um problema sanitário da maior relevância, em praticamente todos os países do mundo.

Agrupadas, muitas vezes, como doença única, em razão da similaridade de suas manifestações clínicas, elas compreendem entidades mórbidas bem conhecidas e distintas, quanto à etiologia, epidemiologia, evolução, prognóstico e profilaxia. Embora, recentemente, novos vírus tenham sido isolados e, em algum momento, associados a hepatites ${ }^{(1,2)}$ tem-se como certa, a existência de cinco tipos de hepatites virais, de importância médica.

De acordo com seu mecanismo habitual de transmissão, as hepatites virais são comumente classificadas em dois grandes grupos: o primeiro corresponde àquelas cuja transmissão se faz pelas vias fecal e oral, englobando as hepatites A e E; no segundo, situam-se as que se transmitem através de outros mecanismos - predominando a via parenteral -representadas pelas hepatites B, C e Delta.

A de conhecimento mais antigo é a hepatite A, por muito tempo conhecida como hepatite infecciosa, de cuja existência já se sabia em meados do século $\mathrm{XVIII}^{(3)}$. Considerando-se que sua transmissão ocorre pelas vias fecal e oral, essa doença tem alta prevalência em regiões onde é precário o saneamento ambiental, o que cria condições propícias para sua disseminação. Essa característica faz com que a hepatite A seja amplamente encontrada no Brasil, apesar de evidências de que a sua transmissão já não acontece tão precocemente quanto em décadas passadas, quando a quase totalidade das crianças tornava-se infectada até os 5 anos de idade ${ }^{(4)}$.

Interessante notar que, não obstante ter sua existência reconhecida desde longa data, o isolamento do vírus da hepatite A (VHA) deu-se apenas em $1973^{(5)}$, lançando a base para o desenvolvimento de uma vacina de elevado poder imunogênico, que 
se tornou disponível na segunda metade da década de 1990.

$\mathrm{O}$ vírus da hepatite $\mathrm{E}$ (VHE), reconhecido também recentemente ${ }^{(6)}$, ocorre em numerosos países em desenvolvimento, onde tem sido associado à epidemias transmitidas por água contaminada com resíduos de esgoto ${ }^{(7)}$. Acomete adultos jovens e apresenta elevada letalidade em mulheres grávidas, atingindo valores próximos de $20 \%$ nas epidemias ${ }^{(8)}$. Excluída essa situação, a hepatite E não costuma associar-se a casos graves, uma vez que, a exemplo da hepatite A, não tem potencial de cronificação. Estudos recentes demonstram a presença do VHE em populações brasileiras, embora a carência de investigações sobre o tema deixe ainda muitas lacunas no conhecimento da história natural dessa doença no Brasil ${ }^{(9,10)}$.

Em 1977, na Itália, Rizzeto et al. descreveram um novo sistema antígenoanticorpo, associado ao vírus da hepatite B (VHB), detectado por imunofluorescência direta apenas no núcleo de células hepáticas de pacientes HBsAg positivos, com doença hepática crônica ${ }^{(11)}$. O achado conduziu à descoberta de um novo tipo da doença, a hepatite Delta, e ao desenvolvimento de técnicas laboratoriais, que permitem detectar anticorpos anti-Delta em indivíduos infecta$\operatorname{dos}^{(12)}$. Pela sua associação obrigatória com a hepatite $\mathrm{B}$, largamente disseminada em extensas regiões do território brasileiro - particularmente na Região Amazônica - e pelo grande potencial de gravidade clínica, esse novo tipo de hepatite reveste-se de grande importância no quadro sanitário, nacional ${ }^{(13,14)}$.

Contudo, das cinco hepatites virais conhecidas, as mais importantes para a saúde pública são, inquestionavelmente, as causadas pelo VHB e pelo VHC (vírus da hepatite $\mathrm{C}$ ). Isso se deve à combinação de dois fatores, um de natureza epidemiológica e outro de natureza clínica. Epidemiologicamente, a relevância dessas doenças deve-se à larga distribuição geográfica e ao enorme número de indivíduos infectados, em praticamente todos os países do mundo. Do ponto de vista clínico, ambas apresentam elevado potencial de cronificação, estando intimamente associadas ao aparecimento de graves afecções hepáticas, destacando-se a cirrose e o carcinoma ${ }^{(7)}$.

Embora, por muito tempo, se soubesse da existência de uma hepatite de transmissão parenteral, então conhecida como hepatite "não-A, não-B", em função de apresentar resultado negativo para os marcadores das hepatites A e B, somente em 1989 chegou- se à identificação do VHC, através do isolamento de um fragmento de DNA derivado do genoma viral de um caso de hepatite não-A, não-B transmitida pela via transfusional ${ }^{(15)}$. O rápido desenvolvimento de um marcador sorológico, específico para o VHC permitiu caracterizá-lo como o mais importante agente causador das hepatites não-A, não-B ${ }^{(16)}$, possibilitando um avanço extraordinário do conhecimento da história natural dessa forma de hepatite.

Assim, estima-se que, nos Estados Unidos, nas últimas três décadas, cerca de 4 milhões de pessoas tenham sido infectadas, com o número de casos novos mantendo-se, atualmente, em torno de 30 mil por ano $^{(17)}$. Para o mundo como um todo, a Organização Mundial da Saúde (OMS) estima a existência de cerca de 170 milhões de portadores crônicos, fato que tem levado as autoridades de saúde pública a considerar a hepatite $\mathrm{C}$ como a grande pandemia do século $\mathrm{XXI}^{(7)}$. Para ilustrar o impacto dessa doença sobre as condições sanitárias e organização dos serviços de saúde, basta citar que as complicações da hepatite $\mathrm{C}$ crônica representam já a primeira causa de transplante hepático, em muitos países do mundo ${ }^{(7)}$.

A transmissão da hepatite $C$ faz-se essencialmente por contato com sangue e hemoderivados contaminados com o vírus, colocando sob máximo risco os usuários de drogas ilícitas, injetáveis, os politransfundidos, os pacientes submetidos à hemodiálise e os indivíduos que sofrem acidente perfurocortante com material contaminado ${ }^{(17 / 20)}$. A possibilidade de transmissão sexual do VHC é ainda questão aberta; embora constantemente aceita como muito menos provável do que a transmissão do VHB ${ }^{(21 / 27)}$, tem sido apontada como uma possibilidade concreta, em publicações recentes ${ }^{(28 / 32)}$. Portanto, o risco de infecção pelo VHC tende a ser mais elevado em grupos populacionais específicos, tais como os indivíduos intensamente expostos à atividade sexual com múltiplos parceiros e a drogas ilícitas, injetáveis, bem como seus respectivos contactantes imediatos ${ }^{(17,18,20,26,28)}$. Em determinadas circunstâncias, esses fatores de risco ocorrem simultaneamente, refletindo maior freqüência de contato com drogas entre aqueles com intensa exposição sexual, múltipla ou entre seus parceiros ${ }^{(33)}$.

A hepatite B permanece como um dos mais graves problemas de saúde pública em muitos países, apesar de, em anos recentes, a vacinação específica e as campanhas educativas contra a aids terem contribuído para uma tendência de redução da sua pre- 
valência em âmbito mundial. A despeito das dificuldades relacionadas à subnotificação dos casos - mesmo em países com tradição de qualidade em serviços de saúde - sabe-se que a hepatite B apresenta distribuição universal e variada, segundo regiões e grupos populacionais. Estima-se que cerca de 300 milhões a 350 milhões de pessoas - aproximadamente 5\% da população mundial - sejam portadoras crônicas do $\mathrm{VHB}^{(34)}$, dos quais 60 milhões deverão morrer devido ao hepatocarcinoma e 45 milhões pelas conseqüências da cirrose hepática ${ }^{(7)}$. Tais projeções conferem a esse vírus uma extraordinária relevância medico-sanitária, sendo apontado pela OMS como o segundo agente carcinogênico conhecido, atrás apenas do tabaco, em termos quantitativos ${ }^{(35)}$.

Reportando-se a uma investigação anterior sobre aspectos epidemiológicos da hepatite $\mathrm{B}$, no município de Cássia dos Coqueiros, SP ${ }^{(36)}$, considera-se importante a inclusão, neste trabalho, de um breve resumo sobre aspectos históricos e evolutivos dessa doença.

A história da identificação da hepatite B, como entidade nosológica própria, parece remontar a 1885. De acordo com o relato de Lürman ${ }^{(37)}$, citado por Gardner ${ }^{(3)}$, ocorreu, naquele ano, uma epidemia de icterícia, que acometeu 191 dos 1289 trabalhadores da construção naval da cidade de Bremen, na Alemanha, que haviam sido vacinados contra a varíola com linfa humana, glicerinada. No mesmo período, nenhum caso de icterícia ocorreu entre 500 outros trabalhadores vacinados com fonte diversa de linfa, enquanto casos esporádicos foram verificados na população geral.

Já no início do século XX, é possível que os arsenicais injetáveis para o tratamento da sífilis tenham contribuído para a disseminação da hepatite $\mathrm{B}$, através de seringas e agulhas contaminadas. Mais recentemente, novas práticas médicas ampliaram, de modo considerável, o risco de infecção, quer pelo contato generalizado com instrumentos potencialmente contaminados, quer pelo risco adicional, representado pelo próprio sangue humano ou seus derivados, largamente utilizados em numerosos procedimentos médicos.

O ponto de partida para o conhecimento científico da hepatite B ocorreu em 1961, quando Allison \& Blumberg descreveram, no soro de politransfundidos, o encontro de anticorpos precipitantes ${ }^{(38)}$. Em 1964, estudando o polimorfismo de proteínas séricas e o desenvolvimento de isoprecipitinas, Blumberg verifi- cou que a proteína contra a qual esses anticorpos reagiam aparentava ser incomum em populações de origem ocidental, porém aparecia com relativa freqüência em indivíduos originários da Micronésia, do Vietnã e de Formosa, e, também, nos aborígenes da Austrália $^{(39)}$. Em 1965, na cidade de Nova York, detectou-se, no soro de um hemofílico, um anticorpo que reagia em presença do soro de um aborígene australiano. Esse achado conduziu à identificação do $\mathrm{VHB}^{(40)}$. O material antigênico do aborígene passou a ser chamado "antígeno Austrália", logo associado a pacientes leucêmicos ${ }^{(40,41,42)}$ e a portadores da síndrome de Down ${ }^{(41,42)}$ e de hepatite ${ }^{(41 / 45)}$. Estudos, que permitiram desvendar a estrutura do VHB, mostraram que o antígeno Austrália corresponde ao envelope viral, sendo, atualmente, designado como antígeno de superfície ou HBsAg.

A descoberta do VHBabriu caminho para o avanço espetacular do conhecimento de uma doença sobre a qual pouco se sabia até então. A importância dessa descoberta pode ser avaliada pela sua enorme repercussão nos meios científicos de todo o mundo, culminando com a concessão do Prêmio Nobel de Medicina a Blumberg.

No estudo do quadro clínico, etiologia e prevenção das hepatites, em geral, e da hepatite $B$, em particular, não podem ser omitidos os trabalhos de Krugman e colaboradores, nas décadas de 1950 e $1960^{(46,47,48)}$. Numa sequiência de experimentos realizados numa instituição para crianças deficientes mentais, onde a incidência de hepatite se mostrava bastante elevada (Willowbrook School, no Estado de Nova York), os autores conseguiram demonstrar a existência de dois tipos de hepatites, que diferiam entre si do ponto de vista clínico, epidemiológico e imunológico. Considerados como trabalhos clássicos, que trouxeram grande contribuição ao entendimento das hepatites A e B, podem também ser apontados como exemplos eloqüentes de desrespeito a princípios éticos, básicos, em experimentações com seres humanos. Todas as suas conclusões - indubitavelmente de valor na elucidação de muitos aspectos, até então ignorados, da história natural das hepatites virais - foram obtidas através da administração deliberada de materiais orgânicos, contendo o VHA e o VHB, a crianças com retardo mental.

Embora a transmissão parenteral tenha se tornado conhecida desde os primórdios das investigações sobre a hepatite $\mathrm{B}^{(46 / 49)}$, pouco se sabia, no final da 
década de 1960, sobre outros possíveis mecanismos capazes de manter o VHB numa comunidade.

Na década de 1970, uma intensa busca do HBsAg, em diferentes fluidos corpóreos e em grupos populacionais distintos, permitiu que se levantassem várias hipóteses sobre mecanismos de transmissão, diversos da via parenteral. Logo no início dessa década, resultados conflitantes sobre a presença do antígeno em fezes e urina ensejaram especulações sobre um eventual envolvimento dessas excreções na transmissão não parenteral do $\mathrm{VHB}^{(50 / 55)}$. Também dessa época, é a busca do antígeno em bile, suco gástrico e lágrimas, com resultado negativo em todos eles ${ }^{(56,57)}$. Positividade para o vírus, entretanto, foi detectada no leite materno ${ }^{(58,59)}$, levando, já em 1974, à recomendação de que mães HBsAg positivas deveriam ser orientadas a não amamentar seus filhos ${ }^{(58)}$.

$\mathrm{O}$ encontro do VHB na saliva, embora com percentuais variáveis, mostrou-se constante em diferentes investigações ${ }^{(55,57,60,61,62)}$, levando à hipótese de transmissão através de beijos com contato boca a boca, de uso coletivo de goma de mascar entre crianças, e até mesmo de tosse ou de espirros ${ }^{(55)}$. A presença do VHB na saliva constituía uma possível explicação para o achado de infectados em agrupamentos familiares, geralmente quando um dos pais era portador crônico do antígeno ${ }^{(63,64)}$. Por outro lado, permitia também especular sobre a transmissão pela via sexual, que passou a ganhar corpo a partir do achado do antígeno no sangue menstrual ${ }^{(65)}$ e nas secreções vaginais $^{(66)}$.

A possibilidade de que a hepatite B pudesse ser uma doença sexualmente transmissível (DST) viria a ser reforçada com o isolamento do antígeno no sê$\operatorname{men}^{(60,61)}$ e com as primeiras evidências de que contactantes íntimos de portadores crônicos pareciam estar sob risco mais elevado de adquirir a infecção ${ }^{67 /}$ 71).

Todos esses achados apontavam, de maneira quase unânime, para a transmissão do VHB pela via sexual, motivando os pesquisadores a buscar a confirmação dessa hipótese, através do estudo de subgrupos populacionais com alta exposição a múltiplos parceiros. A despeito de uma publicação de 1974 não ter evidenciado diferença entre as proporções de prostitutas e freiras colombianas infectadas ${ }^{(72)}$, a hipótese de transmissão pela via sexual acabou por se fortificar, a partir de investigações, envolvendo profissionais do sexo $^{(73)}$ e populações de homossexuais masculinos, citados como de alta promiscuidade, em publicação de
1975, os quais chegavam a um número médio de mil parceiros ao longo da vida ${ }^{(74)}$. Todavia, merece ser mencionado que, até o início da década de 1990, existiam dúvidas, na literatura, acerca do maior risco de transmissão do VHB em populações de prostitutas ${ }^{(75)}$.

Sabe-se, hoje, que a transmissão do VHB pode ser horizontal ou vertical, entendendo-se esta como a passagem do vírus diretamente da mãe para o recémnascido $^{(76)}$, usualmente no parto ou através do contato íntimo que se estabelece depois, e não por troca de sangue ao longo da gestação ${ }^{(7)}$. A importância da transmissão vertical reside no aumento significativo do risco de desenvolvimento de formas crônicas, que é inversamente proporcional à idade em que se adquire a infecção( $0^{(7)}$.

Já a transmissão horizontal decorre do contato com fluidos orgânicos, contendo o VHB, de modo especial com sangue, sêmen e saliva. Abre-se, assim, um amplo leque de possibilidades de transmissão através de vários mecanismos, o que explica, ao menos parcialmente, a vasta distribuição do VHB em diversas partes do mundo, bem como a existência de muitos e diferentes grupos de pessoas com maior risco de adquirir a infecção. Justifica-se, dessa maneira, a maior prevalência da doença entre indivíduos submetidos a determinados riscos, entre os quais se destacam: hemodiálise; multitransfusão de sangue ou hemoderivados; trabalho com sangue humano; acidente perfurocortante com material contaminado; convivência íntima com infectados; asseio pessoal insatisfatório; uso de drogas ilícitas injetáveis; exposição a múltiplos parceiros sexuais.

Entretanto, mesmo com todo o conhecimento acumulado nas últimas décadas sobre as hepatites virais, é de se notar que permanecem, ainda, lacunas importantes sobre a epidemiologia dessas doenças. Ilustrativo disso é a constatação de que, mesmo com anamneses extremamente cuidadosas, não se identificam fatores de risco conhecidos em percentuais consideráveis de indivíduos com quadros crônicos, chegando esse desconhecimento a valores aproximadamente iguais a $30 \%$ para hepatite B e $10 \%$ para hepatite $\mathrm{C}^{(77)}$. Esse fato demonstra, de modo eloqüente, que existe ainda um longo caminho a ser trilhado para que se chegue a atingir um conhecimento pleno sobre a epidemiologia dessas viroses. Por essa razão, impõe-se a continuidade de investigações epidemiológicas de campo, com o objetivo de elucidar aspectos obscuros acerca dos mecanismos de transmissão desses vírus, na natureza. 
PASSOS ADC. Epidemiological aspects of viral hepatitis. Medicina, Ribeirão Preto, 36: 30-36, jan./ march, 2003.

ABSTRACT: Some of the main epidemiological characteristics of the five identified types of viral hepatitis are presented, emphasizing those caused by virus $B$ and $C$. These two forms show a large geographical distribution and ability to cause chronic infections, which are associated with cirrhosis and liver cancer. Thus, these forms of hepatitis are an important public health problem worldwide. Their epidemiological relevance and main transmission routes are discussed.

UNITERMS: Hepatitis, Viral, Human Hepatitis. Epidemiology.

\section{REFERÊNCIAS BIBLIOGRÁFICAS}

1 - HUANG YH; WU JC; LIN CC; SHENG WY; LEE PC; WANG YJ; CHANG FY \& LEE SD. Prevalence and risk factor analysis of TTV infection in prostitutes. J Med Virol 60: 393-395, 2000.

2 - HINRICHSEN H; LEIMENSTOLL G; STEGEN G; SCHRADER H; FOLSCH UR \& SCHMIDT WE. Prevalence of and risk factors for hepatitis G (HGV) infection in haemodialysis patients: A muticentre study. Nephrol Dial Transplant 17: 271-275, 2002.

3 - GARDNER HT. A note on the history of epidemic viral hepatitis in Germany. Am J Med 8: 561-564, 1950.

4 - VITRAL CL; YOSHIDA CF; LEMOS ER;TEIXEIRA CS \& GASPAR, AM. Age-specific prevalence of antibodies to hepatitis $A$ in children and adolescents from Rio de Janeiro, Brazil, 1978 and 1995. Relationship of prevalence to environmental factors. Mem Inst Oswaldo Cruz 93: 1-5, 1998.

5 - FEINSTONE SM; KAPIKIAN AZ \& PURCELI RH. Hepatitis A: detection by immune electron microscopy of a viruslike antigen associated with acute illness. Science 182: 10261028, 1973.

6 - REYES GR; PURDY MA; KIM JP; LUK KC; YOUNG LM; FRY KE \& BRADLEY DW. Isolation of a CDNA from the virus responsible for enterically transmitted non-A, non-B hepatitis. Science 247: 1335-1339, 1990.

7 - SHERLOCK S \& DOOLEY J. Diseases of the liver and biliary system, $10^{\text {th }}$ ed, Blackwell Science, London, 1997.

8 - BRADLEY DW. Enterically-transmitted non-A, non-B hepatitis. Br Med Bull 46: 442-461, 1990.

9 - FOCACCIA R; SETTE H JUNIOR \& CONCEIÇÃO OJ. Hepatitis E in Brazil. Lancet 346:1165, 1995.

10 - TRINTA KS; LIBERTO MI; DE PAULA VS; YOSHIDA CF \& GASPAR AM. Hepatitis E virus infection in selected Brazilian populations. Mem Inst Oswaldo Cruz 96: 25-29, 2001.

11 - RIZZETTO M; CANESE MG; ARICO S; CRIVELLI O; TREPO C; BONINO F \& VERME G. Immunofluorescence detection of new antigen-antibody system (delta/anti-delta) associated to hepatitis B virus in liver and in serum of HBsAg carriers. Gut 18: 997-1003, 1977.

12 - RIZZETTO M. The delta agent. Hepatology 3: 729-737, 1983.
13 - BENSABATH G; HADLER SC; SOARES MC; FIELDS H; DIAS LB; POPPER H \& MAYNARD JE. Hepatitis delta virus infection and Labrea hepatitis. Prevalence and role in fulminant hepatitis in the Amazon Basin. JAMA 258: 479-483, 1987.

14 - BENSABATH G \& DIAS LB. Labrea hepatitis (Labrea black fever) and other fulminant forms of hepatitis in Sena Madureira, Acre, and Boca do Acre, Amazonas, Brasil. Rev Inst Med Trop São Paulo 25: 182-194, 1983.

15 - CHOO QL; KUO G; WEINER AJ; OVERBY LR; BRADLEY DW \& HOUGHTON M. Isolation of a cDNA clone derived from a blood-borne non-A, non-B viral hepatitis genome. Science 244: 359-362, 1989.

16 - CHOO QL; WEINER AJ; OVERBY LR; KUO G; HOUGHTON M \& BRADLEY DW. Hepatitis $C$ virus: The major causative agent of viral non-A, non-B hepatitis. Br Med Bull 46: 423-441, 1990.

17 - ALTER MJ. Epidemiology of hepatitis C. Hepatology 26: 62S65S, 1997. Suppl.1

18 - DE CARVALHO HB; MESQUITA F; MASSAD E; BUENO RC; LOPES GT; RUIZ MA \& BURATTINI MN. HIV and infections of similar transmission patterns in a drug injectors community of Santos, Brazil. Acquir Immun Defic Syndr Hum Retrovirol 12: 84-92, 1996.

19 - THOMAS DL; CANNON RO; SHAPIRO CN; HOOK EW $3^{\text {RD; }}$ ALTER MJ \& QUINN TC. Hepatitis C, Hepatitis B and human immunodeficiency virus infections among non-intravenous drug-using patients attending clinics for sexually transmitted diseases. J Infect Dis 169: 990-995, 1994.

20 - GUADAGNINO V; ZIMATORE G; IZZI A; CAROLEO B; ROCCA A; MONTESANO F; COSTA C; MASCIARI R; NASO E \& BISELLI $R$. Relevance of intravenous cocaine use in relation to prevalence of HIV, hepatitis $B$ and $C$ virus markers among intravenous drug abusers in southern Italy. J Clin Lab Immunol 47: 1-9, 1995.

21 - ORDUNA A; BRATOS MA; GUTIERREZ P; ALMARAZ A; EIROS JM; MARTIN JF; GONZALEZ JM; CARO-PATON A \& RODRIGUEZ-TORRES A. Infection by hepatitis $B$ and $C$ virus in non-intravenous drug using female prostitutes in Spain. Eur J Epidemiol 8: 656-9, 1992.

22 - LEE SD; CHAN CY; WANG YJ; WU JC; LAI KH; TSAI YT \& LO KJ.. Seroepidemiology of hepatitis $C$ infection in Taiwan. Hepatology 13: 380-383, 1991. 
23 - GUTIERREZ P; ORDUNA A; BRATOS MA; EIROS JM; GONZALEZ JM; ALMARAZ A; CARO-PATON A \& RODRIGUEZ-TORRES A. Prevalence of anti-hepatitis $C$ virus antibodies in positive FTA-ABS non-drug abusing female prostitutes in Spain. Sex Transm Dis 19: 39-40, 1992.

24 - SCHOUB BD; JOHNSON S; McANERNEY JM \& BLACKBURN NK. The role of sexual transmission in the epidemiology of hepatitis $C$ virus in black South Africans. Trans R Soc Trop Med Hyg 86: 431-433, 1992.

25 - SANCHEZ-QUIJANO A; REY C; AGUADO I; PINEDA JA; PEREZ-ROMERO M; TORRES Y; LEAL M \& LISSEN E. Hepatitis $C$ virus infection in sexually promiscuous groups. Eur $\mathbf{J}$ Clin Microbiol Infect Dis 9: 610-612, 1990.

26 - LUKSAMIJARULKUL P \& DEANGBUBPHA A. Hepatitis C antibody prevalence and risk factors of some female sex workers in Thailand. Southeast Asian J Trop Med Public Health 28: 507-512, 1997.

27 - OSELLA AR; MASSA MA; JOEKES S; BLANCH N; YACCI MR; CERTONZE S \& SILEONI S. Hepatitis $B$ and $C$ virus sexual transmission among homosexual men. Am J Gastroenterol 93: 49-52, 1998.

28 - MESQUITA PE; GRANATO CF \& CASTELO A. Risk factors associated with hepatitis $C$ virus (HCV) infection among prostitutes and their clients in the city of Santos, São Paulo State, Brazil. J Med Virol 51: 338-343, 1997.

29 - NAKASHIMA K; KASHIWAGI S; HAYASHI J; URABE K; MINAMI $K$ \& MAEDA $Y$. Prevalence of hepatitis $C$ virus infection among female prostitutes in Fukuoka, Japan. J Gastroenterol 31: 664-668, 1996.

30 - NDIMBIE OK; KINGSLEY LA;NEDJAR S \& RINALDO CR. Hepatitis $C$ virus infection in a male homosexual cohort: risk factor analysis. Genitourin Med 72: 213-216, 1996.

31 - HERSHOW RC; KALISH LA; SHA B; TILL M \& COHEN M. Hepatitis $C$ virus infection in Chicago women with or at risk for HIV infection: evidence for sexual transmission. Sex Transm Dis 25: 527-532, 1998.

32 - PINEDA JA; RIVERO A; REY C; HERNANDEZ-QUERO J; VERGARA A; MUÑOZ J; AGUADO I; SANTOS J; TORRONTERAS R \& GALLARDO JA. Association between hepatitis $C$ virus seroreactivity and HIV infection in nonintravenous drug abusing prostitutes. Eur J Clin Microbiol Infect Dis 14: 460-464,1995.

33 - COPPOLA RC; MASIA G; DI MARTINO ML; CARBONI G; MUGGIANU E; PIRO R \& MANCONI PE. Sexual behaviour and multiple infections in drug abusers. Eur $\mathbf{J}$ Epidemiol 12: 429-435, 1996.

34 - SHAPIRO CN \& Margolis HS. Hepatitis B epidemiology and prevention. Epidemiol Rev 12: 221-227, 1990.

35 - WORLD HEALTH ORGANIZATION. Report of a WHO meeting prevention of liver cancer. Geneve: WHO Tech Rep Ser: 69: 1997

36 - PASSOS ADC. Estudo epidemiológico sobre hepatite B em Cássia dos Coqueiros, SP, Brasil. Tese de Doutorado, Faculdade de Medicina de Ribeirão Preto da USP, Ribeirão Preto. p. 1-207, 1991.

37 - LÜRMAN A. Eine Ikterusepidemie. Berl Klin Wchnschr 22: 20, 1885.
38 - ALLISON AC \& BLUMBERG BS. An isoprecipitation reaction distinguishing human serum-protein types. Lancet 1: 634637, 1961.

39 - BLUMBERG BS. Polymorphisms on the serum proteins and the development of iso-precipitins in transfused patients. Bull N Y Acad Med 40: 377-386, 1964.

40 - BLUMBERG BS; ALTER HJ \& VISNICH S. A "new" antigen in leukemia sera. JAMA 191: 541-546, 1965.

41 - BLUMBERG BS; GERSTLEY BJS; HUNGERFORD DA; LONDON WT \& SUTNICK AI. A serum antigen (Australia antigen) in Down's syndrome, leukemia and hepatitis. Ann Intern Med 66: 924-931, 1967.

42 - BLUMBERG BS; SUTNICK AI \& LONDON WT. Hepatitis and leukemia: their relation to Australia antigen. Bull N Y Acad Med 44: 1566-1586, 1968.

43 - PRINCE AM. An antigen detected in the blood during the incubation period of serum hepatitis. Proc Natl Acad Sci USA 60: 814-821, 1968.

44 - COSSART YE. Australia antigen and hepatitis: A review. J Clin Pathol 24: 394-403, 1971.

45 - OKOCHI K \& MURAKAMI S. Observations on Australia antigen in japanese. Vox Sang 15: 374-385, 1968.

46 - KRUGMAN S; WARD R \& GILES JP. The natural history of infectious hepatitis. Am J Med 32: 717-728,1962.

47 - KRUGMAN S; GILES JP \& HAMMOND J. Infectious hepatitis. Evidence for two distinctive clinical, epidemiological and immunological types of infection. JAMA 200: 365-3673, 1967.

48 - KRUGMAN S \& GILES JP. Viral hepatitis. New light on an old disease. JAMA 212:1019-1029, 1970.

49 - ZMUNESS W; PRINCE AM; BROTMAN B \& HIRSCH RL. Hepatitis $B$ antigen and antibody in blood donors: an epidemiologic study. J Infect Dis 127: 17-25, 1973.

50 - GROB PJ \& JEMELKA H. Faecal S.H.(Australia) antigen in acute hepatitis. Lancet 1: 206-208, 1971.

51 - GROB PJ \& JEMELKA HI. Fecal SH-antigen in acute hepatitis. Am J Dis Child 123: 400-401, 1972.

52 - GUST ID; KALDOR J \& NASTASI M. Absence of Au antigen in faeces of patients with Au-positive sera. Lancet 1: 797, 1971.

53 - BLAINEY JD; EARLE A; FLEWETT TH \& WILLIAMS LKL. Is the urine infective in serum hepatitis? Lancet 1: 797, 1971.

54 - TRIPATZIS I. Australia antigen in urine and feces. Am J Dis Child 128: 401-404, 1972.

55 - VILLAREJOS VM; VISONÁ KA; GUTIÉRREZ A \& RODRÍGUES A. Role of saliva, urine and feces in the transmission of type B hepatitis. N Engl J Med 291: 1375-1378, 1974.

56 - AKDAMAR KA; MAUMUS L; CHERRIE EPPS A; LEACH R \& WARREN SSH. Antigen in bile. Lancet 1: 909, 1971.

57 - VITTAL SB; DOURDOUREKAS D \& STEIGMAN F. Hepatitis-B antigen in saliva, urine and tears. Am J Gastroenterol 61: 133-135, 1974.

58 - LINNEMANN CC \& GOLDBERG S. HbAg in breast milk. Lancet 2: 155, 1974. 
59 - BOXALL EH; FLEWETT TH; DANE DS; CAMERON CH; MACCALLUM FO \& LEE TW. Hepatitis B surface antigen in breast milk. Lancet 2: 1007-1008, 1974.

60 - HEATHCOTE J; CAMERON CH \& DANE DS. Hepatitis B antigen in saliva and semen. Lancet 1: 71-73, 1974.

61 - LINNEMANN CC \& GOLDBERG S. Hepatitis-B antigen in saliva and semen. Lancet 1: 320, 1974.

62 - WARD R; WRIGHT A; BORCHERT P \& KLINE E. Hepatitis B antigen in saliva and mouth washings. Lancet 2: 726-727, 1972.

63 - HEATHCOTE J; GATEAU P \& SHERLOCK S. Role of hepatitis$B$ antigen carriers in non-parenteral transmission of the hepatitis-B virus. Lancet 2: 370-371, 1974.

64 - SZMUNESS W; PRINCE AM; HIRSCH RL \& BROTMAN B. Familial clustering of hepatitis B infection. N Engl J Med 289: 1162-1166, 1973.

65 - MAZZUR S. Menstrual blood as a vehicle of Australia-antigen transmission. Lancet 1: 379, 1973.

66 - DARANI M \& GERBER M. Hepatitis-B antigen in vaginal secretions. Lancet 2: 1008, 1974.

67 - HERSH T; MELNICK JL; GOYAL RK \& HOLLINGER FB. Nonparenteral transmission of viral hepatitis type $B$ (Australia antigen-associated serum hepatitis). N Engl J Med 285: 1363-1364, 1971.

68 - HEATHCOTE J \& SHERLOCK S. Spread of acute type-B hepatitis in London. Lancet 1: 1468-1470, 1973.

69 - JEFFRIES DJ; JAMES WH; JEFFERISS FJG; MACLEOD KG \& WILLCOX RR. Australia (hepatitis-associated) antigen in patients attending a venereal disease clinic. Br Med J 2: 455-456, 1973.
70 - WRIGHT RA. Hepatitis B and the HBsAg carrier. An outbreak related to sexual contact. JAMA 232: 717-721, 1975.

71 - FULFORD KWM \& DANE DS. Australia antigen and antibody among patients attending a clinic for sexually transmitted diseases. Lancet 1: 1470-1473, 1973.

72 - ADAM E; HOLLINGER B; MELNICK JL; DUEÑAS A \& RAWIS WE. Type $B$ hepatitis antigen and antibody among prostitutes and nuns: a study of possible venereal transmission. J Infect Dis 129: 317-321, 1974.

73 - PAPAEVANGELOU G; TRICHOPOULOS D; KREMASTINOU T \& PAPOUTSAKIS G. Prevalence of hepatitis $B$ antigen and antibody in prostitutes. Br Med J 2: 256-258, 1974.

74 - SZMUNESS W; MUCH I; PRINCE AM; HOOFNAGLE JH; CHERUBIN CE; HARLEY EJ \& BLOCK GH. On the role of sexual behavior in the spread of hepatitis B infection. Ann Intern Med 83: 489-495, 1975.

75 - HYAMS KC; PHILLIPS IA; TEJADA A; LI O; HERMOZA P; LOPEZ F; ALVA P; CHAUCA G; SANCHEZ S \& WIGNALL S. Hepatitis $B$ in a highly active prostitute population: evidence for a low risk of chronic antigenemia. J Infect Dis 162: 295298, 1990.

76 - GROB P. Introduction to epidemiology and risk of hepatitis B. Vaccine 13: S14-5,1995. Suppl. 1

77 - CENTERS FOR DISEASE CONTROL AND PREVENTION (CDC). Viral hepatitis. Disponível em: http://www.cdc.gov/ncidod/ diseases/hepatitis/slideset. Acesso em 27/01/2003.

Recebido para publicação em 07/02/2003

Aprovado para publicação em 31/03/2003 